PROCEEDINGS OF THE

AMERICAN MATHEMATICAL SOCIETY

Volume 130, Number 4, Pages 1145-1151

S 0002-9939(01)06133-0

Article electronically published on September 19, 2001

\title{
ON THE VOLUME AND THE GAUSS MAP IMAGE OF SPACELIKE HYPERSURFACES IN DE SITTER SPACE
}

\author{
JUAN A. ALEDO AND LUIS J. ALÍAS
}

(Communicated by Wolfgang Ziller)

\begin{abstract}
In this paper we prove that a complete spacelike hypersurface $M^{n}$ in de Sitter space such that its image under the Gauss map is contained in a hyperbolic geodesic ball of radius $\varrho$ is necessarily compact and its $n$ dimensional volume satisfies $\omega_{n} / \cosh (\varrho) \leq \operatorname{vol}(M) \leq \omega_{n} \cosh ^{n}(\varrho)$, where $\omega_{n}$ denotes the volume of a unitary round $n$-sphere. We also characterize the case where these inequalities become equalities. As an application of our result, we also conclude that Goddard's conjecture is true under the assumption that the hyperbolic image of the hypersurface is bounded.
\end{abstract}

\section{INTRODUCTION}

Let $\mathbf{L}^{n+2}$ be the $(n+2)$-dimensional Lorentz-Minkowski space, that is, the real vector space $\mathbf{R}^{n+2}$ endowed with the Lorentzian metric tensor $\langle$,$\rangle given by$

$$
\langle v, w\rangle=\sum_{i=1}^{n+1} v_{i} w_{i}-v_{n+2} w_{n+2},
$$

and let $\mathbf{S}_{1}^{n+1} \subset \mathbf{L}^{n+2}$ be the $(n+1)$-dimensional unitary de Sitter space, that is,

$$
\mathbf{S}_{1}^{n+1}=\left\{x \in \mathbf{L}^{n+2}:\langle x, x\rangle=1\right\} .
$$

As is well-known, for $n \geq 2$ the de Sitter space $\mathbf{S}_{1}^{n+1}$ is the standard simply connected Lorentzian space form of positive constant sectional curvature. A smooth immersion $\psi: M^{n} \longrightarrow \mathbf{S}_{1}^{n+1} \subset \mathbf{L}^{n+2}$ of an $n$-dimensional connected manifold $M^{n}$ is said to be a spacelike hypersurface if the induced metric via $\psi$ is a Riemannian metric on $M^{n}$. As is usual, the spacelike hypersurface is said to be complete if the Riemannian induced metric is a complete metric on $M^{n}$.

The interest in the study of spacelike hypersurfaces in de Sitter space is motivated by the fact that such hypersurfaces exhibit nice Bernstein-type properties. In 1977 Goddard [7] conjectured that the only complete spacelike hypersurfaces with constant mean curvature in de Sitter space should be the totally umbilical ones. This conjecture, which turned out to be false in this original statement, motivated

Received by the editors April 17, 2000 and, in revised form, October 5, 2000.

2000 Mathematics Subject Classification. Primary 53C42; Secondary 53B30, 53C50.

Key words and phrases. de Sitter space, spacelike hypersurface, volume, Gauss map, hyperbolic image, mean curvature.

This work has been partially supported by DGICYT Grant No PB97-0784-C03-02 and Consejería de Educación y Cultura CARM, Programa Séneca (PRIDTYC).

(C)2001 American Mathematical Society 
the work of an impressive number of authors who considered the problem of characterizing the totally umbilical spacelike hypersurfaces of de Sitter space in terms of some appropriate geometric assumptions. In particular, Akutagawa [2] showed that Goddard's conjecture is true if the constant mean curvature $H$ of the hypersurface satisfies $0 \leq H^{2} \leq 1$ when $n=2$ and $0 \leq H^{2}<4(n-1) / n^{2}$ in the general case $n \geq 3$. As an application of it, Akutagawa also proved that when $n=2$ Goddard's conjecture is also true under the additional hypothesis of the compactness of the surface (see also [13] for a simultaneous and independent alternative proof of these facts given by Ramanathan when $n=2$ ). In [10], Montiel extended this last result to the general case by showing that the only compact spacelike hypersurfaces in de Sitter space are the totally umbilical round spheres.

More recently, Cheng and Ishikawa 6 have shown that the totally umbilical round spheres are the only compact spacelike hypersurfaces in de Sitter space with constant scalar curvature $S<n(n-1)$. Li [9] and Zheng [14, 15] also obtained interesting characterizations of these hypersurfaces under the hypothesis of constant scalar curvature. In 4 the authors, jointly with Romero, have recently found some other characterizations of the totally umbilical round spheres in de Sitter space as the only compact spacelike hypersurfaces with constant higher order mean curvature, under appropriate hypotheses.

Observe that $(0, \ldots, 0,0,1) \in \mathbf{L}^{n+2}$ is a unit timelike vector field globally defined on $\mathbf{L}^{n+2}$, which determines a time-orientation on $\mathbf{L}^{n+2}$. Thus we can choose a unique timelike unit normal field $N$ on $M^{n}$ which is future-directed in $\mathbf{L}^{n+2}$, and hence we may assume that $M^{n}$ is oriented by $N$. By parallel translation to the origin in $\mathbf{L}^{n+2}$, we can regard the field $N$ as a map $N: M^{n} \longrightarrow \mathbf{H}^{n+1}$, where $\mathbf{H}^{n+1}$ denotes the $(n+1)$-dimensional hyperbolic space, that is,

$$
\mathbf{H}^{n+1}=\left\{x \in \mathbf{L}^{n+2}:\langle x, x\rangle=-1, \quad x_{n+2} \geq 1\right\} .
$$

We will refer to the map $N: M^{n} \longrightarrow \mathbf{H}^{n+1}$ as the future-directed Gauss map of $M^{n}$, and the image $N(M)$ will be called the hyperbolic image of the hypersurface. In this paper we prove the following result.

Theorem. Let $\psi: M^{n} \longrightarrow \mathbf{S}_{1}^{n+1} \subset \mathbf{L}^{n+2}$ be a complete spacelike hypersurface in de Sitter space such that its hyperbolic image is bounded. Then $M^{n}$ is diffeomorphic to a sphere $\mathbf{S}^{n}$. In particular, $M^{n}$ is compact and its $n$-dimensional volume satisfies the following inequalities:

$$
\operatorname{vol}(M) \leq \omega_{n} \cosh ^{n}(\varrho)
$$

and

$$
\operatorname{vol}(M) \geq \frac{\omega_{n}}{\cosh (\varrho)}
$$

where $\varrho$ stands for the radius of a geodesic ball in $\mathbf{H}^{n+1}$ containing the hyperbolic image of $M^{n}$, and $\omega_{n}$ denotes the volume of a round $n$-sphere of radius one. Moreover, (1) becomes an equality if and only if $M^{n}$ is a totally umbilical round sphere of radius $\cosh (\varrho)$, while (2) becomes an equality if and only if $M^{n}$ is a totally geodesic round sphere (of radius one, $\varrho=0$ ).

Our proof depends on the fact that if the hyperbolic image of $M^{n}$ is contained in a hyperbolic geodesic ball centered at a point $a \in \mathbf{H}^{n+1}$, then the projection of $M^{n}$ onto the sphere $\left\{x \in \mathbf{S}_{1}^{n+1}:\langle a, x\rangle=0\right\}$ is a diffeomorphism (see the next section for the details). As an application of our result, we also conclude that 
Goddard's conjecture is true if the hyperbolic image of the spacelike hypersurface is bounded (see Corollary 1). The corresponding result for the case of spacelike hypersurfaces in the Lorentz-Minkowski space $\mathbf{L}^{n+1}$ states that spacelike hyperplanes are the only complete spacelike hypersurfaces with constant mean curvature in $\mathbf{L}^{n+1}$ whose hyperbolic image is bounded. This theorem was proved simultaneous and independently by Aiyama [1] and Xin [12 (see also 11] for a previous weaker version of this result given by Palmer).

As another application of our result, we also observe that the pinching result given by the second author in [5] for compact spacelike hypersurfaces can be extended to the case of complete hypersurfaces (see Corollary 22).

\section{Proof of the Theorem}

Let us assume that the hyperbolic image of $M^{n}$ is contained in a geodesic ball $\tilde{B}(a, \varrho)$ in $\mathbf{H}^{n+1}$ of radius $\varrho>0$ centered at the point $a \in \mathbf{H}^{n+1}$. Recall that

$$
\tilde{B}(a, \varrho)=\left\{q \in \mathbf{H}^{n+1}: 1 \leq-\langle a, q\rangle \leq \cosh (\varrho)\right\},
$$

so that

$$
1 \leq-\langle a, N\rangle \leq \cosh (\varrho)
$$

on $M^{n}$.

Observe that the intersection of $\mathbf{S}_{1}^{n+1} \subset \mathbf{L}^{n+2}$ and the spacelike hyperplane $a^{\perp}=\left\{x \in \mathbf{L}^{n+2}:\langle a, x\rangle=0\right\}$ defines a round $n$-sphere of radius one, which is a totally geodesic hypersurface in $\mathbf{S}_{1}^{n+1}$. We will refer to that $n$-sphere as the equator of $\mathbf{S}_{1}^{n+1}$ determined by $a$. We will see then that $M^{n}$ is diffeomorphic to an $n$-sphere by showing that its projection onto the equator determined by $a$

$$
\mathbf{S}_{a}^{n}=\left\{x \in \mathbf{S}_{1}^{n+1}:\langle a, x\rangle=0\right\}
$$

is a diffeomorphism between $M^{n}$ and $\mathbf{S}_{a}^{n}$. In fact, the projection $\pi: \mathbf{S}_{1}^{n+1} \longrightarrow \mathbf{S}_{a}^{n}$ of $\mathbf{S}_{1}^{n+1}$ onto the equator $\mathbf{S}_{a}^{n}$ is given by

$$
\pi(x)=\frac{1}{\sqrt{1+\langle a, x\rangle^{2}}}(x+\langle a, x\rangle a), \quad x \in \mathbf{S}_{1}^{n+1},
$$

so that the projection of $M^{n}$ onto $\mathbf{S}_{a}^{n}$ is the composition map $\Pi=\pi \circ \psi: M^{n} \longrightarrow \mathbf{S}_{a}^{n}$ written as

$$
\Pi(p)=\frac{1}{\sqrt{1+\langle a, \psi(p)\rangle^{2}}}(\psi(p)+\langle a, \psi(p)\rangle a), \quad p \in M^{n} .
$$

A straightforward computation shows that

$$
d \Pi(X)=\frac{1}{\sqrt{1+\langle a, \psi\rangle^{2}}} X+\frac{1}{\left(1+\langle a, \psi\rangle^{2}\right)^{3 / 2}}(-\langle a, \psi\rangle\langle a, X\rangle \psi+\langle a, X\rangle a)
$$

for every tangent vector field $X \in \mathcal{X}(M)$. Therefore,

$$
\Pi^{*}\left(\langle,\rangle \mathbf{S}_{a}^{n}\right)=\frac{1}{1+\langle a, \psi\rangle^{2}}\langle,\rangle+\frac{\langle\nabla\langle a, \psi\rangle,\rangle\langle\nabla\langle a, \psi\rangle,\rangle}{\left(1+\langle a, \psi\rangle^{2}\right)^{2}} \geq \frac{1}{1+\langle a, \psi\rangle^{2}}\langle,\rangle,
$$

where $\nabla\langle a, \psi\rangle$ is the gradient of $\langle a, \psi\rangle$ on $M^{n}$, and $\langle,\rangle_{\mathbf{S}_{a}^{n}}$ denotes the metric on $\mathbf{S}_{a}^{n}$. 
Recall that $\nabla\langle a, \psi\rangle=a^{\top}$, where $a^{\top}$ denotes the component of $a$ which is tangent to $M^{n}$, that is,

$$
a=a^{\top}-\langle a, N\rangle N+\langle a, \psi\rangle \psi
$$

In particular,

$$
1+\langle a, \psi\rangle^{2}=\langle a, N\rangle^{2}-\left|a^{\top}\right|^{2} \leq\langle a, N\rangle^{2} \leq \cosh ^{2}(\varrho)
$$

on $M^{n}$, so that

$$
\Pi^{*}\left(\langle,\rangle_{\mathbf{S}_{a}^{n}}\right) \geq \frac{1}{1+\langle a, \psi\rangle^{2}}\langle,\rangle \geq \frac{1}{\langle a, N\rangle^{2}}\langle,\rangle \geq \frac{1}{\cosh ^{2}(\varrho)}\langle,\rangle
$$

From (6) it follows that $\Pi$ is a local diffeomorphism. Since $\langle$,$\rangle is a com-$ plete Riemannian metric on $M^{n}$, the same holds for the homothetic metric $\widetilde{\langle,\rangle}=$ $\cosh ^{-2}(\varrho)\langle$,$\rangle . Then, equation (9) means that the map$

$$
\Pi:\left(M^{n}, \widetilde{\langle,\rangle}\right) \longrightarrow\left(\mathbf{S}_{a}^{n},\langle,\rangle \mathbf{S}_{a}^{n}\right)
$$

increases the distance. If a map, from a connected complete Riemannian manifold $M_{1}$ into another connected Riemannian manifold $M_{2}$ of the same dimension, increases the distance, then it is a covering map and $M_{2}$ is complete [8, Chapter VIII, Lemma 8.1]. Hence $\Pi$ is a covering map, but $\mathbf{S}_{a}^{n}$ being simply connected this means that $\Pi$ is in fact a global diffeomorphism between $M^{n}$ and the equator $\mathbf{S}_{a}^{n}$. This finishes the proof of the first assertion in our Theorem.

As for the volume of $M^{n}$, using the diffeomorphism $\Pi: M^{n} \longrightarrow \mathbf{S}_{a}^{n}$ we know that

$$
\omega_{n}=\operatorname{vol}\left(\mathbf{S}_{a}^{n}\right)=\int_{\mathbf{S}_{a}^{n}} \mathrm{~d} S=\int_{M} \Pi^{*}(\mathrm{~d} S),
$$

where $\mathrm{d} S$ stands for the $n$-dimensional volume element of the round sphere $\mathbf{S}_{a}^{n}$ with respect to the orientation induced by its future-directed Gauss map a. Using (4) and (5) it follows that

$$
\begin{aligned}
\Pi^{*}(\mathrm{~d} S)\left(X_{1}, \ldots, X_{n}\right) & =\operatorname{det}\left(d \Pi\left(X_{1}\right), \ldots, d \Pi\left(X_{n}\right), a, \Pi\right) \\
& =\frac{1}{\left(1+\langle a, \psi\rangle^{2}\right)^{(n+1) / 2}} \operatorname{det}\left(X_{1}, \ldots, X_{n}, a, \psi\right) \\
& =\frac{-\langle a, N\rangle}{\left(1+\langle a, \psi\rangle^{2}\right)^{(n+1) / 2}} \operatorname{det}\left(X_{1}, \ldots, X_{n}, N, \psi\right),
\end{aligned}
$$

for tangent vector fields $X_{1}, \ldots, X_{n} \in \mathcal{X}(M)$. In other words,

$$
\Pi^{*}(\mathrm{~d} S)=\frac{-\langle a, N\rangle}{\left(1+\langle a, \psi\rangle^{2}\right)^{(n+1) / 2}} \mathrm{~d} V,
$$

where $\mathrm{d} V$ is the $n$-dimensional volume element of $M^{n}$ with respect to the induced metric and the orientation given by its future-directed Gauss map $N$, so that

$$
\omega_{n}=\int_{M} \frac{-\langle a, N\rangle}{\left(1+\langle a, \psi\rangle^{2}\right)^{(n+1) / 2}} \mathrm{~d} V .
$$

From (3) and (7) we know that

$$
1 \leq-\langle a, N\rangle=\sqrt{1+\langle a, \psi\rangle^{2}+\left|a^{\top}\right|^{2}},
$$


and therefore

$$
\begin{aligned}
\frac{-\langle a, N\rangle}{\left(1+\langle a, \psi\rangle^{2}\right)^{(n+1) / 2}} & =\frac{\sqrt{1+\langle a, \psi\rangle^{2}+\left|a^{\top}\right|^{2}}}{\left(1+\langle a, \psi\rangle^{2}\right)^{(n+1) / 2}} \geq \frac{\sqrt{1+\langle a, \psi\rangle^{2}}}{\left(1+\langle a, \psi\rangle^{2}\right)^{(n+1) / 2}} \\
& =\frac{1}{\left(1+\langle a, \psi\rangle^{2}\right)^{n / 2}} .
\end{aligned}
$$

Now using (8) we conclude from (10) that

$$
\omega_{n} \geq \int_{M} \frac{1}{\left(1+\langle a, \psi\rangle^{2}\right)^{n / 2}} \mathrm{~d} V \geq \int_{M} \frac{1}{\cosh ^{n}(\varrho)} \mathrm{d} V=\frac{\operatorname{vol}(M)}{\cosh ^{n}(\varrho)},
$$

which means that inequality (1) holds. Moreover, this inequality becomes an equality if and only if

$$
1+\langle a, \psi\rangle^{2}=\langle a, N\rangle^{2}=\cosh ^{2}(\varrho)
$$

that is, if and only if $\langle a, \psi\rangle= \pm \sinh (\varrho)$ is constant on $M^{n}$ and $\psi(M)$ is the totally umbilical round $n$-sphere of radius $\cosh (\varrho)$ defined by

$$
\psi(M)=\left\{x \in \mathbf{S}_{1}^{n+1}:\langle a, x\rangle= \pm \sinh (\varrho)\right\} .
$$

On the other hand, using now that

$$
\frac{-\langle a, N\rangle}{\left(1+\langle a, \psi\rangle^{2}\right)^{(n+1) / 2}} \leq \frac{\cosh (\varrho)}{\left(1+\langle a, \psi\rangle^{2}\right)^{(n+1) / 2}} \leq \cosh (\varrho),
$$

we obtain from (10) that

$$
\omega_{n} \leq \int_{M} \cosh (\varrho) \mathrm{d} V=\cosh (\varrho) \operatorname{vol}(M),
$$

and inequality (2) holds. Moreover, equality holds here if and only if

$$
1+\langle a, \psi\rangle^{2}=\langle a, N\rangle^{2}=1,
$$

which means that $\langle a, \psi\rangle=0$ on $M^{n}$ and $\psi(M)$ is the totally geodesic round $n$-sphere of radius one defined by

$$
\psi(M)=\left\{x \in \mathbf{S}_{1}^{n+1}:\langle a, x\rangle=0\right\} .
$$

\section{Applichtions AND Remarks}

As a first interesting consequence of our result we can state the following result, which means that Goddard's conjecture is true under the assumption that the hyperbolic image of the hypersurface is bounded.

Corollary 1. The only complete spacelike hypersurfaces with constant mean curvature in de Sitter space whose hyperbolic image is bounded are the totally umbilical round spheres.

Proof. Let $\psi: M^{n} \longrightarrow \mathbf{S}_{1}^{n+1} \subset \mathbf{L}^{n+2}$ be a complete spacelike hypersurface with constant mean curvature in de Sitter space such that its hyperbolic image is bounded. We know from our theorem that $M^{n}$ is compact, and by Montiel's theorem we can conclude that $M^{n}$ is a totally umbilical round sphere. Actually this can be easily seen by observing that for $a \in \mathbf{H}^{n+1}$, the function $u=\langle a, N\rangle-H\langle a, \psi\rangle$ defined on $M^{n}$ satisfies

$$
\Delta u=\langle a, N\rangle\left(\operatorname{trace}\left(A^{2}\right)-n H^{2}\right) .
$$


Here $A$ stands for the shape operator associated to the future-directed Gauss map $N, H=-\operatorname{trace}(A) / n$ defines the mean curvature of the hypersurface (which is assumed to be constant), and $\Delta$ denotes the Laplacian operator on $M^{n}$. In fact, by the Cauchy-Schwarz inequality it follows that $\operatorname{trace}\left(A^{2}\right)-n H^{2} \geq 0$ with equality at umbilical points. Since $\langle a, N\rangle \leq-1<0$, equation (11) implies that $\Delta u \leq 0$ on $M^{n}$, which is compact, so that $\Delta u \equiv 0$ and $M^{n}$ is a totally umbilical round sphere in de Sitter space.

In [5], the second named author has recently shown that if the hyperbolic image of a compact spacelike hypersurface $M^{n}$ is contained in a geodesic ball in $\mathbf{H}^{n+1}$ of radius $\varrho>0$ and the Ricci curvature of $M^{n}$ satisfies the pinching condition

$$
\text { Ric } \leq \frac{n-1}{\cosh ^{2}(\varrho)}
$$

then $M^{n}$ must be a totally umbilical round sphere of radius $\cosh (\varrho)$. The proof of this result was a consequence of an integral formula involving the Ricci curvature and the scalar curvature of the hypersurface. As another application of our Theorem, we remark here that this pinching result can be extended to the case of complete hypersurfaces in the following way.

Corollary 2. Let $M^{n}$ be an n-dimensional complete spacelike hypersurface in $\mathbf{S}_{1}^{n+1}$ such that its hyperbolic image is contained in a geodesic ball in $\mathbf{H}^{n+1}$ of radius $\varrho$. If the Ricci curvature of $M^{n}$ satisfies Ric $\leq(n-1) / \cosh ^{2}(\varrho)$, then $M^{n}$ must be a totally umbilical round sphere of radius $\cosh (\varrho)$.

Finally, let $\psi: M^{n} \longrightarrow \mathbf{S}_{1}^{n+1} \subset \mathbf{L}^{n+2}$ be a spacelike hypersurface in de Sitter space. We will say that the hypersurface itself is timelike bounded in $\mathbf{S}_{1}^{n+1}$ if there exists a unit timelike vector $a \in \mathbf{H}^{n+1}$ such that the function $\langle a, \psi\rangle$ is bounded on $M^{n}$. Observe that, by equation (8), if the hyperbolic image of $M^{n}$ is bounded, then $M^{n}$ is timelike bounded. It follows from the proof of our Theorem that the following result also holds true.

Corollary 3. Every complete spacelike hypersurface in de Sitter space which is timelike bounded is compact.

In particular, the only complete spacelike hypersurfaces with constant mean curvature in de Sitter space which are timelike bounded are the totally umbilical round spheres. The corresponding result for the case of spacelike hypersurfaces in the Lorentz-Minkowski space has been recently obtained by the authors in [3], and it states that the only complete spacelike hypersurfaces with constant mean curvature in the Lorentz-Minkowski space which are bounded between two parallel hyperplanes are the totally geodesic spacelike hyperplanes.

\section{REFERENCES}

[1] R. Aiyama, On the Gauss map of complete space-like hypersurfaces of constant mean curvature in Minkowski space, Tsukuba J. Math. 16 (1992), 353-361. MR 93m:53062

[2] K. Akutagawa, On spacelike hypersurfaces with constant mean curvature in the de Sitter space, Math. Z. 196 (1987), 13-19. MR 88h:53052

[3] J.A. Aledo and L.J. Alías, On the curvatures of bounded complete spacelike hypersurfaces in the Lorentz-Minkowski space, Manuscripta Math. 101 (2000), 401-413. MR 2001a:53091

[4] J.A. Aledo, L.J. Alías and A. Romero, Integral formulas for compact spacelike hypersurfaces in de Sitter space. Applications to the case of constant higher order mean curvature, J. Geom. Phys. 31 (1999), 195-208. MR 2000e:53092 
[5] L.J. Alías, On the Ricci curvature of compact spacelike hypersurfaces in de Sitter space, Geom. Dedicata 77 (1999), 297-304. MR 2000h:53087

[6] Q-M. Cheng and S. Ishikawa, Spacelike hypersurfaces with constant scalar curvature, Manuscripta Math. 95 (1998), 499-505. MR 99e:53087

[7] A.J. Goddard, Some remarks on the existence of spacelike hypersurfaces of constant mean curvature, Math. Proc. Cambridge Phil. Soc. 82 (1977), 489-495. MR 56:16547

[8] S. Kobayashi and K. Nomizu, Foundations of Differential Geometry, Vol. II, Interscience, New York, 1969. MR 38:6501

[9] H. Li Global rigidity theorems of hypersurfaces, Ark. Mat. 35 (1997), 327-351. MR 98j:53074

[10] S. Montiel, An integral inequality for compact spacelike hypersurfaces in de Sitter space and applications to the case of constant mean curvature, Indiana Univ. Math. J. 37 (1988), 909917. MR 90c:53167

[11] B. Palmer, The Gauss map of a spacelike constant mean curvature hypersurface of Minkowski space, Comment. Math. Helv. 65 (1990), 52-57. MR 91a:53014

[12] Y.L. Xin, On the Gauss image of a spacelike hypersurface with constant mean curvature in Minkowski space, Comment. Math. Helv. 66 (1991), 590-598. MR 93d:53016

[13] J. Ramanathan, Complete spacelike hypersurfaces of constant mean curvature in de Sitter space, Indiana Univ. Math. J. 36 (1987), 349-359. MR 88g:53067

[14] Y. Zheng, On spacelike hypersurfaces in the de Sitter space, Ann. Global Anal. Geom. 13 (1995), 317-321. MR 96j:53062

[15] Y. Zheng, Spacelike hypersurfaces with constant scalar curvature in the de Sitter spaces, Differ. Geom. Appl. 6 (1996), 51-54. MR 97e:53124

Departamento de Matemáticas, Universidad de Castilla-La Mancha, Escuela Politécnica Superior de Albacete, 02071 Albacete, Spain

E-mail address: jaledo@pol-ab.uclm.es

Departamento de Matemáticas, Universidad de Murcia, 30100 Espinardo, Murcia, SPAIN

E-mail address: ljalias@um.es 